\title{
Hip 3D Joint Mechanics Analysis of Normal and Obese Individuals' Gait
}

\author{
M.H. Mazlan1, N.A. Abu Osman2, and W.A.B. Wan Abas2 \\ 1 Department of Electrical and Electronics Engineering, University Tun Hussein Onn Malaysia, Batu Pahat, Malaysia \\ 2 Department of Biomedical Engineering, University of Malaya, Kuala Lumpur, Malaysia
}

\section{INTRODUCTION}

3D joint moments, angle and joint power vectors have initially

been used to investigate the gait strategy used in

normal adult's and children's gait [1][2]. However, the

discovery pertaining to this issue is still new and most of the

time the research is focused on normal subjects and during

level walking activity. Thus, the area of the research seems

can be expanded to the other form of motion activities and

involve with obese community which believed associated

with a large number of loads cycle [3].

Theoretically, the joint power is the best method to appropriately

describe the muscle activities whether it is negative,

null or positive joint power which corresponds to an

absorbed, null or generated energy coarsely associated to

eccentric, isometric or concentric muscular actions [4].

However, when stand alone it seems insufficient to give

details explanation on the joint and muscular activities.

Therefore, the introduction of 3D joint angle is purposely to

help the 3D joint power interpretations in a manner of describing

the proportion of the joint moment which contributes

to the movements (i.e. propels, resists or stabilized the

joint). Moreover, with the aid of inverse dynamics approach,

the calculations are become simpler without the

need of musculo-skeletal modeling as been required in the

forward dynamics analysis.

The focused of the study was to analyze the hip 3D joint

mechanics for obese and normal individuals during stair

ascending activity. It could be used to investigate the gait 
strategy used and how the neuromuscular adaptations applied to the joint. It is of interest due its vast contributions in understanding the mechanics of normal and pathology of the hip joint during normal and abnormal loading conditions [5]. Besides the human hip joint can withstands peak contact forces up to 4 to 5 times of the body weight [6][7][8], however, the repetition of high joint loading profile will make it more susceptible to injury and structural deterioration over time and this situations can be worsen when involving with obese individuals.

In the present study, the selection of stair ascending rather than stair descending activity was made based on the judgment that, even both hip joint contact forces and moments are significantly higher for stair descending than for stair ascending activity, however, the effective contact areas of the stair ascending are relatively small in comparison to descending stair activity which lead to the high pressure distribution at the hip joint acetabulum (even with a small value of joint contact forces) [6][9]. In addition, for obese individuals, this task is quite demanding where the motor functions are reduced [10]. Besides, this task is comparably much more important than level walking activity due to during level walking activity the hip joint angular velocity remains relatively small and passive resistance of the skeleton to the gravity is considerably less than one times of the body weight [7].

Human body automatically has been programmed to adapt to the outside environment for the sake of the skeletal health of the joint. For obese individuals, the compensatory mechanism relative to their BMI (body mass index) including slower walking speed, shorter stride length, increased double support phase and decreased knee range of motion are the true examples of the kinematics adaptations of human locomotion [6][11][12][13]. This phenomena are associated with an adaptation to minimize metabolic energy expended per unit distance traveled [8], to reduce peak pressure distributions [5], to offset total inertia 
generated [14][15] and above all to reduce muscle forces and moment [11][16][17][18]. Therefore, at the end of the day it is believed that this study will give different perspectives in describing the mechanics adaptations of a body in reaction to internal and external mechanical changes.

In this context, the hypothesis is that, it is hard to describe the difference of the gait strategy used between the obese and normal individuals due to the effect of the mechanics adaptations of the joint. Therefore, with the help of $3 \mathrm{D}$ joint angle interpretations, it is believed that, at the end of the day these phenomena can be successfully described and investigated.

Full text is available at :

http://download.springer.com/static/pdf/845/chp\%253A10.1007\%252F978-3-642-217296 43.pdf?auth66=1393724075 e5f7595bca07a8c2fb6b12704beba604\&ext=.pdf

http://link.springer.com/chapter/10.1007/978-3-642-21729-6_43 